\title{
Ejes temáticos y palabras clave en la Comunicación para el Desarrollo y el Cambio Social en España. Análisis de marcadores lingüísticos en revistas españolas de comunicación
}

\author{
Gonzalo Ceballos-Castro \\ Universidad de Cádiz \\ Facultad de Ciencias Sociales y de \\ la Comunicación \\ Avda. de la Universidad s/n \\ 11405 Jerez de la Frontera \\ E-mail: gonzalo.ceballos@uca.es
}

EJES TEMÁTICOS Y PALABRAS CLAVE EN LA COMUNICACIÓN PARA EL DESARROLLO Y EL CAMBIO SOCIAL EN ESPAÑA. ANÁLISIS DE MARCADORES LINGÜISTICOS EN REVISTAS ESPAÑOLAS DE COMUNICACIÓN

RESUMEN: El objetivo de esta investigación es indicar aquellas temáticas que hacen posible la rigurosa delimita ción del campo de la Comunicación para el Desarrollo y el Cambio Social (CDCS), frente a otros subcampos de la investigación en comunicación limitrofes, afines y, al tiempo, diferentes, como son la Comunicación Política, el Marketing Social o la Educación para el Desarrollo. A partir de la estrategia metodológica del análisis de contenido y la lin güistica de corpus hemos analizado, dentro de la producción académica en revistas españolas de comunicación en el período 2000-2015, los marcadores temáticos que caracterizan al discurso de la CDCS frente al de las áreas limí trofes indicadas. Los resultados muestran la centralidad que en la CDCS tienen los siguientes elementos: 1) la investigación de carácter participativo; 2) lo comunitario y alternativo, en concreto las emisoras de radio comunitarias y 3) la cuestión de la alternatividad.

PALABRAS Claves: enfoque cientifico; comunicación; desarrollo; cambio social; lingüistica de corpus.

SUMARIO: 1. Introducción y aproximación teórica. 2. Materiales y métodos. 3. Resultados y análisis. 3.1. El campo de la comunicación. 3.2. La sociedad, su organización y la participación ciudadana. 3.3. El cambio y la transformación social. 4. Conclusiones.
MARTA SÁNChez-SAUS LASERNA

Universidad de Cádiz

Facultad de Filosofia y Letras

Avda. Dr. Gómez Ulla s/n

11003 Cádiz

E-mail: marta.sanchezsaus@uca.es

THEMATIC AXES AND KEYWORDS IN COMMUNICATION FOR DEVELOPMENT AND SOCIAL CHANGE IN SPAIN. ANALYSIS OF LINGUISTIC MARKERS IN SPANISH COMMUNICATION JOURNALS

ABSTRACT: The objective of this research is to indicate which topics make possible the rigorous delimitation of the field of Communication for Developmen and Social Change (CDSC), compared to other subfields that are bordering or re lated to it and, at the same time, different, such as Political Communication, Social Marketing or Education for Development. Based on the methodologica strategy of content analysis and corpus linguistics, we have analyzed, within the academic production in Spanish communication journals in the period 2000 2015 , the thematic markers that characterize the discourse of the CDSC compared to that of the bordering areas in dicated. The results show the centrality in CDSC of the following elements: 1 ) participatory research; 2) the community and alternative perspective, in particular the community radio stations, and 3) the issue of the alternativity.

KEY WORDS: scientific approach; social change; communication; development; corpus linguistics.

SUMMARY: 1 . Introduction and theoretical approach. 2. Materials and methods. 3. Results and analysis. 3.1. The field of communication. 3.2. The society, its organization and citizen participation. 3.3. The change and the social transformation. 4. Conclusions.
VíCTOR MANUEL MARÍ-SÁEZ

Universidad de Cádiz

Facultad de Ciencias Sociales y de la Comunicación

Avda. de la Universidad s/n

11405 Jerez de la Frontera

E-mail: victor.mari@uca.es

\section{AXES THEMATIQUES ET MOTS CLES DANS LA COMMUNICATION POUR LE DEVELOPPEMENT ET LE CHANGEMENT SOCIAL EN ESPAGNE. ANALYSE DES MARQUEURS LINGUISTIQUES DANS LES JOURNAUX DE COMMUNICATION ESPAGNOLS}

RÉSUMÉ: L'objectif de cette recherche est d'indiquer les sujets qui permettent une délimitation rigoureuse du domaine de la communication pour le développement et du changement social (CDCS), par rapport à d'autres sous-domaines de la recherche sur la communication, qu'elle soit limitrophe, différente ou parallèle, comme la communication politique, le marketing social ou éducation pour le développement. Sur la base de la stratégie méthodologique de l'analyse de contenu et de la linguistique de corpus, nous avons analysé, au sein de la production académique de revues de communication espagnoles dans la période 2000-2015, les marqueurs thématiques qui caractérisent le discours du CDCS par rapport à celui des domaines limites indiquées. Les résultats montrent la centralité des éléments suivants dans la CDCS: 1) recherche participative; 2) la communauté et les alternatives, en particulier les stations de radio communautaires, et 3) le problème de l'alternativité.

MOTS CLÉS: approche scientifique; communication ; développement; changement social; linguistique de corpus.

SOMMAIRE: 1. Introduction et approche théorique. 2. Matériels et méthodes. 3. Résultats et analyse. 3.1. Le domaine de la communication 3.2. La société, son organisation et la participation citoyenne. 3.3. Le changement et la transformation sociale. 4. Conclusions.
Fecha de Recepción Fecha de Revisión Fecha de Aceptación Fecha de Publicación
$31 / 09 / 2018$

$07 / 10 / 2018$

$12 / 11 / 2018$

01/12/2018 DOI: http://dx.doi.org/10.25267/Pragmalinguistica.2018.i26.01 


\section{Ejes temáticos y palabras clave en la Comunicación para el Desarrollo y el Cambio Social en España. Análisis de marcadores lingüisticos en revistas españolas de comunicación ${ }^{1}$}

GonZalo Ceballos-CASTRO², MARTa SÁncheZ-Saus Laserna \& Víctor Manuel MARí-SÁEZ

\section{INTRODUCCIÓN Y APROXIMACIÓN TEÓRICA}

La Comunicación para el Desarrollo y el Cambio Social (CDCS) en España ha experimentado en las últimas décadas un incremento de su protagonismo en el campo global de la investigación en comunicación. Este proceso de toma de conciencia de la importancia de la comunicación en el campo de la cooperación y de la acción social por parte de las instituciones, las ONGD y los movimientos sociales viene acompañado de una amplia diversidad de enfoques teórico-prácticos desde los que se concibe la acción comunicativa. La mayoría de estos enfoques están dominados por un imaginario en el que la comunicación se reduce a la transmisión de información y a la modificación de las conductas. En este sentido, este proceso de institucionalización puede ser releído como una implosión del campo, debido a la tardía y precaria consolidación fruto de la insuficiente producción científica de calidad, del desarrollo epistemológico poco riguroso y del escaso avance institucional de este campo (Marí Sáez, 2017).

Según las investigaciones impulsadas en España dirigidas a reconstruir el estado de la cuestión del campo (Coordinadora de ONG para el Desarrollo España [CONGDE], 2005; Chaparro, 2002; Chaves, 2012; Erro, 2003; Fernández Viso, 2012; González, 2007; Jerez et al., 2008; Meda, 2012; Marí Sáez, 2016; Moragas, 2005; Pinazo, D. y Nos, E., 2013; y Santolino, 2010), la CDCS en España ha ido pasando por tres grandes etapas: i) el periodo de los pioneros (1980-1994), ii) la etapa de la emergencia (1994-2002), y iii) la etapa de la institucionalización e implosión (2003-actualidad). Este fenómeno ha traído como consecuencia (Marí-Sáez, V. M. y Ceballos-Castro, G., 2015) la existencia en España de trabajos de investigación realizados desde un enfoque de CDCS más formal que real. Investigaciones que, al obviar las

\footnotetext{
${ }^{1}$ El presente artículo se enmarca dentro del Proyecto de I+D del Ministerio de Economía y Competitividad "Evaluación y monitorización de la Comunicación para el Desarrollo y el Cambio Social en España: diseño de indicadores para la medición de su incidencia social" (EvalComDev) CSO2014-52005-R (2015-2017). IP: Víctor Manuel Marí Sáez (Universidad de Cádiz). Cuenta, asimismo, con el apoyo del Proyecto de I+D del Ministerio de Economía y Competitividad "Comunicación especializada y terminografia: usos terminológicos relacionados con los contenidos y perspectivas actuales de la semántica léxican (FFI2014-54609-P). IP: Miguel Casas Gómez.

${ }^{2}$ Gonzalo Ceballos-Castro es contratado predoctoral de personal investigador en formación (PIF) por la Universidad de Cádiz, convocado por concurso público por Resolución del Rector $\mathrm{UCA} / \mathrm{REC} 02 \mathrm{VI} / 2015$.
} 
perspectivas de análisis, herramientas conceptuales y estrategias comunicativas específicas de este enfoque, se desarrollan en realidad desde áreas limítrofes y afines a esta perspectiva, pero no coincidentes, como la Comunicación Política, la Economía Política de la comunicación, el Marketing social o la Educación para el desarrollo. Esta hibridación y superposición de conceptos y marcos teóricos procedentes de estos campos afines termina por afectar, negativamente, a la correcta delimitación del campo de la CDCS.

En este trabajo consideraremos, siguiendo a Mari (2016) que la comunicación para el desarrollo (Servaes, 2007a, 2007b; Lennie, J. y Tacchi, J., 2010) y la comunicación para el cambio social (Wilkins, 2000; Gumucio, A. y Tufte, Th., 2006) son diferentes aproximaciones para denominar y caracterizar un campo relacionado con el papel de la comunicación en los esfuerzos estratégicos para superar los problemas sociales colectivos. Desde estos autores, la CDCS se concibe desde una doble vertiente, académica y práctica (Gumucio, A. y Tufte, Th., 2006), como praxis, esto es, como "...la reflexión y la acción de los hombres sobre el mundo para transformarlo" (Freire, 1970: 23). En este sentido, la CDCS es una práctica reflexionada y, a la vez, una construcción teórica que conduce necesariamente a la acción (Marí, 2016).

En este contexto, la presente investigación forma parte de un proyecto interdisciplinar cuyo objetivo es la evaluación del campo de la CDCS en España $^{3}$, a partir del diseño de un dispositivo metodológico que, partiendo de distintos tipos de marcadores, permita dibujar un mapa sobre la presencia de la CDCS en la investigación sobre comunicación en España, tomando como referencia investigaciones similares impulsadas con éxito en países anglosajones (Lennie, J. y Tacchi, J., 2013). En concreto, el presente trabajo propone una perspectiva de investigación complementaria a los estudios de carácter historiográfico, bibliométrico y lingüístico realizados hasta la fecha (Marí, 2016; Mari-Sáez, V. M. y Ceballos-Castro, G., 2015; Sánchez-Saus, 2017, 2018). Tomando como estrategia metodológica el análisis de contenido desde la lingüística de corpus, el objetivo de la presente investigación es mostrar aquellas temáticas, líneas de investigación y enfoques que son nucleares y hacen posible la delimitación de investigaciones y reflexiones realizadas desde el enfoque propio de la CDCS.

Para ello, hemos realizado un análisis del discurso académico sobre CDCS frente al de áreas limítrofes, afines y, al tiempo, diferentes a él, como la Comunicación Política, la Economía Política de la comunicación, el Marketing social o la Educación para el desarrollo. En concreto, hemos realizado un análisis de contenido a partir de palabras clave sobre un corpus de artículos científicos afines a esta perspectiva identificados en un estudio bibliométrico precedente (Marí-Sáez, V. M. y Ceballos-Castro, G., 2015). A continuación, hemos comparado estos resultados con las categorías temáticas predominantes en la reflexión académica sobre CDCS detectadas en un estudio precedente desde la lingüística de corpus (Sánchez-Saus, 2017).

\footnotetext{
${ }^{3}$ Proyecto EvalComDev. CSO2014-52005-R (2015-2017).
} 
La razón principal para usar la lingüística de corpus (LC) es que nos permite llevar a cabo análisis de contenido de grandes cantidades de texto con rapidez, precisión y consistencia. Entre las diversas definiciones existentes sobre LC retomamos, por su precisión y rigor, la que proporciona Parodi (2008: 96):

la LC se define, strictu sensu, como una metodología para la investigación de las lenguas y del lenguaje, la cual permite llevar a cabo investigaciones empíricas en contextos auténticos [...]. Desde este enfoque, se estudia información lingüistica original y completa, compilada a través de corpus, dado que desde la LC no se apoya la indagación de datos fragmentados, inconexos o de textos incompletos, sino de unidades de sentido y con propósitos comunicativos específicos.

Mancera y Pano (2014: 306), por su parte, resumen las aplicaciones de la lingüística de corpus señalando que los corpus nos permiten “...una adecuada representación del discurso en muestras amplias y representativas de textos originales; [...] es posible realizar análisis más amplios y detallados; ofrece mayor fiabilidad en los análisis cuantitativos y cualitativos; los resultados son acumulativos y confrontables con posteriores investigaciones."

La LC pone a nuestra disposición numerosas herramientas para llevar a cabo análisis de contenido. De entre ellas, en este artículo empleamos el análisis de palabras clave, ya que,

frente a los análisis de contenido basados en listas de palabras frecuentes, las listas de palabras clave no contienen las más frecuentes, sino aquellas con frecuencias desproporcionadas en relación a la norma. De este modo, los análisis de palabras clave subrayan el cambio o variación y neutralizan las similitudes entre dos corpus (Duque, 2014: 42).

En este sentido, el presente estudio busca poner de relieve lo nuclear del enfoque de la CDCS respecto de otros abordajes y perspectivas teóricas afines con las que comparte muchas de sus temáticas y objetos de estudio. Como señala igualmente este autor, el análisis de palabras clave permite llevar a cabo un análisis de contenido en el que asumimos que hay una “...relación entre las repeticiones de una palabra y su relevancia en el discurso y en la construcción de representaciones" (Duque 2014: 42).

\section{MATERIALES Y MÉTODOS}

El análisis que presentamos en este artículo ha sido realizado a partir de dos corpus académicos, el primero de ellos está compuesto por 216.771 palabras extraídas de los artículos científicos (32 en total) que el análisis bibliométrico llevado a cabo por Marí y Ceballos-Castro (2015) determinó como pertenecientes a áreas afines y limitrofes a la CDCS en España en el periodo 2000-20154 (Ver Anexo). Este corpus está compuesto por aquellos

\footnotetext{
${ }^{4} \mathrm{El}$ estudio de referencia de Marí y Ceballos publicado en 2015 comprende el período 2000 -
} 
artículos que, en el análisis bibliométrico mencionado, se identificaron como aquellos que no reúnen suficientes requisitos para ser incluidos en el campo de la CDCS, pero que, sin embargo, incluyen conceptos y referencias teóricas de otros campos afines y limitrofes como los ya enunciados previamente en este artículo.

El segundo corpus, que nos servirá de referencia para la comparación, está formado por los artículos (24 en total) que el análisis bibliométrico llevado a cabo por Marí y Ceballos-Castro (2015) determinó como pertenecientes al campo de la CDCS en España en el período 2000-20155 (Ver Anexo). Este corpus está compuesto por aquellos artículos que en el análisis bibliométrico mencionado se identificaron como los que sí reúnen suficientes requisitos para ser incluidos en el campo de la CDCS. La delimitación temporal del objeto de estudio (2000-2015) se justifica por las etapas de la CDCS en España ${ }^{6}$. En este sentido, el periodo seleccionado abarca la etapa de la institucionalización e implosión del campo (2003-actualidad) para mostrar, a pesar de la inconsistencia epistemológica a la que hemos hecho referencia, la presencia del enfoque propio de la CDCS en la producción bibliográfica frente a las otras perspectivas afines pero no iguales.

Para el tratamiento de los corpus hemos empleado el programa AntConc (Anthony, 2017). Con las propias opciones que proporciona el programa, ambos corpus han sido lematizados ${ }^{7}$, uniendo bajo una misma forma todas las variantes morfológicas de una palabra, así como las posibles variantes ortográficas. Además, se ha utilizado una stoplist, es decir, una lista de palabras que se excluyen del análisis. Esta lista está compuesta por palabras gramaticales frecuentes en español como preposiciones, conjunciones, determinantes, verbos auxiliares. El análisis no se ha realizado con los textos académicos en bruto, sino con la lista de las 1.000 primeras palabras más frecuentes empleadas en ambos corpus de textos académicos. Para calcular el índice de propensión o keyness de cada palabra clave, el programa AntConc lleva incorporada la medida estadística Log Likelihood ${ }^{8}$. Podemos ver sus valores de significación en la siguiente tabla 1 :

Tabla 1. Valores de significación de Log Likelihood

\begin{tabular}{c} 
95th percentile; $5 \%$ level; $p<0,05 ;$ critical value $=3,84$ \\
\hline 99th percentile; $1 \%$ level; $p<0,01 ;$ critical value $=6,63$ \\
\hline 99.9th percentile; $0,1 \%$ level; $p<0,001 ;$ critical value $=10,83$ \\
\hline Fuente: http: / / ucrel.lancs.ac.uk/llwizard.html.
\end{tabular}

2012. Posteriormente se amplió la investigación hasta abarcar el período 2000-2015.

5 Ver nota 4.

${ }^{6}$ El periodo de los pioneros (1980-1994), la etapa de la emergencia (1994-2002), y la etapa de la institucionalización e implosión (2003-actualidad).

7 Según Gómez Díaz (2005), la lematización es el proceso de eliminación automática de partes no esenciales de los términos (sufijos, prefijos) para reducirlos a su parte esencial (lema) y facilitar la eficacia de la indización y la consiguiente recuperación.

8 Sobre el uso de la medida estadística Log Likelihood para la comparación de corpus, véase Rayson y Garside (2000). 
En la lista de las primeras 150 palabras clave del corpus de los artículos afines que aquí analizamos el valor de la keyness es siempre superior a 230.000, por lo que los valores que presentamos pueden ser considerados, con gran distancia, significativos. Es decir, las palabras clave obtenidas configuran estrictamente aquellas que son significativamente usadas con más frecuencia en los artículos académicos en España. Una vez obtenidas las palabras clave compararemos los resultados con las categorias temáticas generales que caracterizan al discurso de la CDCS (Sánchez-Saus, 2017), para mostrar las temáticas y enfoques centrales y específicos que surgen del corpus académico de los textos identificados como pertenecientes a la CDCS.

Ciertas palabras clave, si tenemos en cuenta el contexto en el que aparecen, solo pueden incluirse en una u otra temática; otras, pueden incluirse en varias pues las categorias no son mutuamente excluyentes. Para ello utilizamos la herramienta KWIC (KeyWords in Context) que proporciona AntConc (Anthony, 2017). Esta herramienta permite observar cómo se han usado las palabras clave en el corpus y rodeadas de qué otras palabras aparecen en su contexto original. Asimismo, el programa nos permite observar la frecuencia de los clusters que nos interesen, esto es, de las combinaciones en las que aparece una palabra determinada. En algunas ocasiones la herramienta KWIC nos va a permitir extraer colocación ${ }^{9}$ de las que forma parte la palabra clave, aunque normalmente nos servirá, simplemente, para observar cómo se combinan sintagmáticamente, es decir, a qué palabras aparecen con frecuencia unidas o muy próximas en el corpus.

\section{RESULTADOS Y ANÁLISIS}

A continuación se muestran los resultados del análisis de los marcadores temáticos que caracterizan al discurso académico sobre la CDCS frente al discurso académico de áreas afines pero diferentes a esta perspectiva con la finalidad de mostrar aquellas temáticas y enfoques que son nucleares y hacen posible la delimitación rigurosa de investigaciones y reflexiones realizadas desde el enfoque propio de la CDCS.

Las 150 primeras palabras clave del corpus académico de las áreas afines empleado aparecen en la Tabla 2 (ver anexo), acompañadas de la frecuencia absoluta y relativa con la que han aparecido y su keyness o índice de propensión. Por otro lado, las 150 primeras palabras clave del corpus académico que realmente es encuadrable en CDCS aparecen en la Tabla 3 (ver anexo), acompañadas de sus valores. Del discurso sobre CDCS (tabla 3) se extraen tres grandes categorias (Sánchez-Saus, 2017), que tienen que ver

\footnotetext{
9 Entendemos el término colocación como aquellas «unidades fraseológicas formadas por dos unidades léxicas en relación sintáctica, que no constituyen, por sí mismas, actos de habla ni enunciados; y que, debido a su fijación en la norma, presentan restricciones de combinación establecidas por el uso, generalmente de base semántica" (Corpas Pastor, 1996: 66).
} 
con 1) la comunicación, 2) la sociedad, su organización y la participación ciudadana y 3) la necesidad del cambio y la transformación sociales. A continuación pasamos a analizar los datos de las tablas precedentes.

\subsection{El CAMPO DE LA COMUNICACIÓN}

Esta categoría reúne un gran número de las palabras clave obtenidas. Por un lado, en el corpus de CDCS encontrábamos "comunicación", "debate" y "estrategia" (unida a "de comunicación" 14 veces). Por otro lado, en el primer corpus de las perspectivas limitrofes respecto a la CDCS estas palabras clave también ocupan posiciones similares de relevancia dentro de las 150 palabras clave, lo cual apunta a que estas temáticas son recurrentes y compartidas por ambos discursos.

En este sentido, vemos cómo estos términos aparecen en el corpus de las perspectivas afines similar número de veces. Términos como "estrategia" unido a "de comunicación" (aparece 22 veces). Igual ocurre con la combinación del término "debate" junto con "público", que aparece 6 veces en los afines y 5 en CDCS, y con "social", que aparece 4 y 1 veces respectivamente. Se observa que este grupo de palabras apunta a ciertas temáticas generales que son compartidas por el enfoque de la CDCS como por las perspectivas que forman parte de las perspectivas afines como la Comunicación politica o la Publicidad social y solidaria.

Por el contrario, el sustantivo "comunicación” lo encontramos en el corpus sobre CDCS combinado con el adjetivo "participativo" 35 veces. Sin embargo, en el corpus de los enfoques afines vemos la combinación en tan solo 2 ocasiones. Si profundizamos en este resultado y nos fijamos en la distribución vemos que esta combinación de términos aparece en 9 de los 24 artículos que forman el corpus de CDCS. Es decir, se distribuye en el 37,5\% de los artículos, mientras que en el corpus de los afines se concentra en tan solo uno de los 32 artículos (3\%) que lo forman. Estos resultados ponen de manifiesto la centralidad que ocupa la articulación y reflexión sobre comunicación y participación dentro del campo de investigación de la CDCS. Es decir, representa una temática nuclear en CDCS y, a la vez, nos da pistas de los limites entre esta perspectiva y los diferentes enfoques analizados fronterizos a esta perspectiva, pero diferentes en sus marcos teóricos, conceptos centrales y palabras clave.

Si nos fijamos ahora en la posición y en los valores del término "participativo" vemos que en el corpus de CDCS aparece en la posición 23, mientras que, por el contrario, en el corpus de los enfoques afines aparece en la posición 363, con una frecuencia relativa $(0,000179913) 4$ veces menor y una keyness (112.255) 4,6 veces menor. Estos valores apuntan a la desviación entre ambos discursos y, a la vez, a la centralidad que hemos observado de esta temática dentro del discurso de la CDCS. 
Siguiendo con el análisis de este primer eje referido a la comunicación, en el corpus de CDCS son también muy numerosas las palabras relacionadas con medios de comunicación: "emisora", "radio", "prensa", "mediático", "medio" que aparece seguido de "masivo" en 22 ocasiones y de "de masas" 79 veces. En el corpus de las perspectivas afines también encontramos, con algo menos de frecuencia, "medio" unido a "masivo" (8 veces) y "de masas" (24 veces). Esto demuestra que tanto para la CDCS como para las perspectivas afines esta temática es central y a la vez compartida en sus investigaciones.

Ambos corpus reflexionan sobre los medios de comunicación masivos pero desde diferentes enfoques como puede ser desde la comunicación política (como forma de incidir sobre la opinión pública), desde el marketing y la publicidad social (como forma de incidir a través de los medios masivos en el comportamiento con fines sociales y solidarios), o desde la Economía Política de la Comunicación (más preocupada por las estructuras económicas de los medios masivos, su configuración, su concentración, etc.). Sin embargo, hay que apuntar que en los enfoques afines términos como "radio" o "emisora" ocupan posiciones muy bajas lo que denota que estas temáticas no son tan recurrentes en su discurso. Vemos como "radio" ocupa la posición 26 en el corpus sobre CDCS frente a la posición 920 que ocupa en las perspectivas afines, con una frecuencia relativa $(0,00035983)$ casi 4 veces menor y una keyness (39.697) 12 veces menor que en el corpus sobre CDCS. Por su parte "emisora" ocupa la posición 30 en el corpus sobre CDCS frente a la posición 1.406 , con una frecuencia relativa $(0,000092263) 8,5$ veces menor y una keyness (22.555) 20,5 veces menor que en el corpus sobre $\mathrm{CDCS}^{10}$.

Como resumen de este primer eje del análisis de los datos se puede decir que en esta primera categoría referida a la comunicación existe entre ambos discursos una convergencia respecto de las temáticas que podemos considerar generales y transversales a los diferentes enfoques fronterizos analizados, como la investigación sobre las estrategias de comunicación, el debate público y social o la investigación y reflexión sobre los medios de comunicación masivos, las radios/emisoras o la prensa. Sin embargo, se observa una diferencia clara entre ambos discursos en lo referente a la articulación entre comunicación y participación, que apunta a la delimitación por parte de las investigaciones que forman el corpus de la CDCS de un área de investigación, actuación y reflexión concreta y específica.

\footnotetext{
10 Esta disparidad que observamos en los valores de estos términos clave se pondrá de relieve en la siguiente categoría referida a la sociedad, su organización y la participación ciudadana. En esta se hace referencia a lo comunitario que articulado con los medios de comunicación hará más patente la diferencia entre los enfoques de las perspectivas analizadas.
} 


\subsection{LA SOCIEDAD, SU ORGANIZACIÓN Y LA PARTICIPACIÓN CIUDADANA}

Pertenecen a este segundo eje de análisis palabras clave como "ciudadanía" y "colectivo". El término "colectivo" está dentro de las 150 primeras palabras clave en ambos corpus, lo que indica que es un término muy usado en ambos discursos. Se aprecia que el término clave "ciudadanía" está en la posición 423 en el corpus de las perspectivas afines, en cierta medida alejada de la posición 113 que ocupa en CDCS. Sin embargo, si observamos sus valores vemos que en el discurso de los enfoques afines la frecuencia relativa $(0,000424411)$ y la keyness $(97.235)$ son tan solo dos veces menor que en el corpus de CDCS. Es decir, el uso de este término dentro del corpus es similar, lo que apunta a que esta palabra clave es muy utilizada en ambos discursos.

Si analizamos conjuntamente los términos "participación" y "ciudadanía" se observa que la combinación "participación ciudadana" aparece 16 veces en el corpus de las perspectivas afines y 14 veces en los artículos académicos sobre CDCS. En la misma línea, si analizamos conjuntamente la presencia de "participación" junto con "social" aparece 8 veces en ambos discursos. Y si combinamos ahora "comunicación" y "sociedad", la combinación "comunicación social" aparece 45 veces en el corpus de los enfoques afines frente a las 15 veces que aparece en la perspectiva de la CDCS. Si nos fijamos en la distribución vemos cómo en CDCS esta combinación se reparte en 5 de los 24 artículos (21\%), mientras que en las perspectivas afines se distribuye en 19 de los 32 (59\%).

Estos resultados apuntan a que la investigación y reflexión sobre participación ciudadana, participación social y comunicación social son temáticas importantes y, a la vez, transversales a ambos discursos, el de la CDCS y el de los enfoques afines a esta perspectiva como el enfoque de Marketing social y la Educación para el desarrollo. Pero, a la vez, estos resultados remarcan lo que hemos observado en la primera categoria, esto es, que la articulación entre comunicación y participación es un eje central en las reflexiones e investigaciones de la CDCS.

El término "movimiento" aparece en la misma posición en ambos corpus (23) y dentro de las primeras posiciones. Estos valores denotan la relevancia de estos términos en ambos discursos. Si combinamos, ahora, "movimiento" y "social", se observa cómo el sintagma aparece 82 veces en el corpus de los denominados afines, y 54 en el discurso de la CDCS. Lo mismo ocurre con la siguiente palabra clave, el término "acción", que aparece en el corpus de CDCS unido a "social" en 9 ocasiones, y a "cooperativa" en 4. Mientras que en el corpus de las perspectivas afines aparece unido a "social" 23 veces, y ninguna a "cooperativa".

Estos resultados ponen de manifiesto que la investigación y reflexión sobre los movimientos sociales y las estrategias de acción social son temáticas centrales y a la vez compartidas por ambos discursos tanto el de la CDCS como el de las perspectivas afines a esta. 
Pero el hecho de que la combinación "acción cooperativa" no aparezca en las investigaciones de los enfoques afines es muy reveladora, en el sentido de que lo cooperativo remite a lo participativo, a lo comunitario y alternativo, temáticas que son nucleares en el campo de CDCS, especialmente en la corriente latinoamericana del campo (Peruzzo, 2008; Uranga, 2009).

El término "comunitario", en el corpus sobre CDCS, ocupa la posición 11, mientras que en el corpus de los enfoques afines y diferentes ocupa la posición 366. Si nos fijamos en sus valores se observa que en el corpus de las perspectivas afines la frecuencia relativa $(0,00018453)$ es 5,5 veces menor y su keyness (111.373) es 6,4 veces menor. Estos valores nos dan pistas de la relevancia de este término en el discurso de la CDCS frente al discurso de los enfoques afines.

En este momento se hace necesario recuperar la reflexión, que apuntábamos en la categoría anterior referida a la comunicación, respecto de los diferentes enfoques a la hora de abordar los medios de comunicación por parte de ambos corpus, el de la CDCS y el de los enfoques limitrofes respecto a esta perspectiva.

En el corpus sobre CDCS el término "comunitario", combinado con la palabra "radio", aparece 32 veces, mientras que el otro corpus de los subcampos limítrofes esta combinación aparece únicamente 2 veces.

Si miramos la distribución vemos que en el corpus sobre CDCS, la combinación "radio" junto con "comunitaria" se reparte en 9 de los 24 artículos que forman el corpus $(37,5 \%)$. Mientras que en los afines se concentra en 2 de los 32 artículos (6,25\%). La combinación "medio" junto con "comunitario" se reparte en CDCS en 6 artículos (25\%), mientras que en los afines se concentra en $3(9,4 \%)$. Si observamos la combinación del término "medio" junto con "alternativo" vemos que esta combinación aparece 24 veces en el discurso de la CDCS, distribuido en 6 de los 24 artículos (25\%), frente a las solo 4 veces que aparece en el discurso de las perspectivas afines, concentrado en 4 de los 32 artículos $(12,5 \%)$.

Con estos datos se puede decir que dentro de este segundo eje de análisis referido a la sociedad, su organización y la participación ciudadana el enfoque de la CDCS tiene entre sus principales temáticas a los medios comunitarios y alternativos. Estos resultados conectan con las investigaciones de un experto del campo como Alfonso Gumucio (2001) cuando afirma que las radios comunitarias representan el ejemplo paradigmático de la puesta en práctica del enfoque participativo de la CDCS. Por el contrario, esta temática y preocupación no la encontramos en el discurso de los enfoques afines a esta perspectiva.

Como resumen de esta categoría referida a la sociedad, su organización y la participación ciudadana, se puede decir que se pone especialmente de manifiesto la afinidad y el solapamiento que existe entre los enfoques que forman parte de ambos corpus. Existe una convergencia en temáticas transversales compartidas, como la reflexión sobre los movimientos sociales y las estrategias de acción social. Sin embargo, también se aprecia con especial 
significación en este segundo eje la centralidad que ocupan en las reflexiones de la CDCS ciertas temáticas relacionadas con la articulación entre la comunicación y lo comunitario y alternativo, y en concreto, la reflexión e investigación sobre las emisoras de radio comunitarias.

\subsection{EL CAMBIO Y LA TRANSFORMACIÓN SOCIAL}

Finalmente, el tercer eje de nuestro análisis apunta a las conexiones entre la comunicación y la transformación social. El término clave "cambio" tiene posiciones dentro de las 150 palabras clave en ambos corpus. Sin embargo, si nos fijamos en sus valores vemos que en las perspectivas afines la frecuencia relativa es $(0,001061028) 3$ veces menor y su keyness (312.580) 4,5 veces menor que en el corpus de la CDCS. Esta diferencia se pone de manifiesto al combinar este término con la palabra clave "social". La combinación aparece 273 veces en CDCS, frente a las 69 veces que aparece en el discurso de las perspectivas afines a esta. Si nos fijamos en la distribución vemos que esta combinación se recoge en 22 de los 24 artículos (91,7\%) que forman el corpus de CDCS. Mientras que en el corpus de los afines aparece concentrado en 16 de los 32 artículos (50\%) que lo forman. Por el contrario, vemos cómo la combinación del término "transformación" junto con "social" aparece 18 veces en la CDCS y 5 veces en el discurso de los afines. Esta combinación no tiene las connotaciones teóricas y epistemológicas que tiene el término "cambio social", lo que justifica su baja presencia y en especial en el discurso de la CDCS.

Siguiendo con la reflexión sobre el cambio social, si observamos la frecuencia de los elementos que conforman el sintagma "comunicación para el desarrollo y el cambio social": vemos que el término "comunicación" combinado con "desarrollo" aparece 170 veces en el corpus de CDCS frente a las 43 veces que aparece en el discurso de los afines. En el corpus sobre CDCS esta combinación aparece distribuida en 15 de los 24 artículos (62,5\%) que conforman el corpus académico. Por el contrario, en el corpus de los afines esta combinación aparece concentrada en 11 de los 32 artículos (34\%) que conforman el corpus. Estos resultados apuntan a la centralidad que ocupa en CDCS la reflexión y articulación entre comunicación, desarrollo y cambio social frente a las investigaciones realizadas desde perspectivas afines a este enfoque.

Por otro lado, la combinación del término "comunicación" junto con "alternativa" aparece 49 veces, frente a las escasas 11 veces que lo encontramos en el discurso de los enfoques afines. La expresión aparece en este segundo discurso distribuida en tan solo 2 de los 32 (6\%) artículos, mientras que aparece en 8 de los 24 (33\%) que componen el corpus de la CDCS. Podemos decir, por tanto, que se pone de relieve la centralidad que existe dentro de la perspectiva de la CDCS la reflexión e investigación sobre la comunicación desde un enfoque participativo y alternativo. 


\section{Conclusiones}

La Comunicación para el Desarrollo y el Cambio Social (CDCS) en España pasa en la actualidad por una fase de institucionalización $y$, a la vez, de implosión del campo, debido a la debilidad epistemológica con la que se ha ido construyendo en sus fases precedentes. En este contexto, con nuestra investigación hemos querido arrojar luz sobre aquellas temáticas, estrategias y enfoques que son nucleares en la producción académica de esta perspectiva y la diferencia respecto de otros abordajes teóricos próximos pero a la vez diferentes. Con este objetivo, de la mano de la metodología de la lingüistica de corpus, hemos llevado a cabo un análisis de las palabras clave que caracterizan la producción académica sobre CDCS frente al discurso académico de áreas limítrofes a esta perspectiva, como la Comunicación Política, la Economía Política de la comunicación, el Marketing social o la Educación para el desarrollo.

$\mathrm{El}$ análisis de las palabras clave nos permite concluir que en un primer eje general sobre comunicación el enfoque participativo es central en las investigaciones impulsadas desde el campo de la CDCS. En una segunda categoría referida a la sociedad, su organización y la participación ciudadana se observa con especial significación que la reflexión e investigación sobre las emisoras de radio comunitarias es lo nuclear en este campo de la investigación. Por último se observa que la reflexión e investigación sobre comunicación, desarrollo y cambio social desde un enfoque participativo y alternativo es constitutivo de esta perspectiva.

Estos resultados, junto con los estudios de carácter historiográfico, bibliométrico y lingüístico precedentes (Marí, 2016; Marí y Ceballos 2015; Sánchez-Saus, 2017, 2018), nos pueden servir para delimitar la implosión aludida, producto del poco rigor teórico y epistemológico desde los que se ha ido construyendo el campo de la CDCS en España. Al tiempo, el análisis y las investigaciones presentadas en este trabajo nos permiten diseccionar el contenido real de los textos, más allá de las filiaciones y pertenencias teóricas que puedan sugerir elementos formales como los títulos, los resúmenes o las palabras claves desde las que se autopresentan las investigaciones analizadas.

\section{REFERENCIAS}

ANTHONY, L. (2017): AntConc (versión 3.4.1) [software informático], Tokyo: Waseda University.

CHAPARRO, M. (2002): Sorprendiendo al futuro. Comunicación para el desarrollo e información audiovisual, Barcelona: Imedea.

CHAVES, I. (coord.) (2012): Comunicación para el cambio social. Universidad, sociedad civil y medios,
Madrid: Los Libros de la Catarata.

COORDINADORA DE ONG PARA EL DESARROLLO ESPAÑA (CONGDE) (2005): Informe de la CONGDE sobre la percepción social de las ONGD: asi nos ven, Madrid: Coordinadora de ONGD de España. 
DUQUE, E. (2014): “Análisis de contenido mediante análisis de palabras clave: La representación de los participantes en los discursos de Esperanza Aguirre", Mediaciones Sociales. Revista de Ciencias Sociales y de la Comunicación, 13, pp. 39-73.

ERRO, J. (2003): Descubrir y construir procesos de comunicación social, Bilbao: Hegoa.

FERNÁNDEZ VISO, A. (2012): "Historia de una travesía inconclusa: la comunicación para el desarrollo y el cambio social en la investigación y la docencia universitarias en España”, Cuadernos de Información y Comunicación, 17, pp. 4162.

FREIRE, P. (1970) Pedagogy of the Oppressed, London: Continuum.

GÓMEZ DÍAZ, R. (2005): La lematización en español: una aplicación para la recuperación de información, Gijón: Ediciones Trea.

GONZÂLEZ, H. (2007): Estrategias de comunicación en las ONG de desarrollo. Departamentos, funciones e impacto en los medios, Madrid: Cideal.

GUMUCIO, A. (2001): Making Waves: stories of participatory communication for social change: a report to the Rockefeller Foundation, Rockefeller Foundation.

GUMUCIO, A. \& Tufte, Th (2006) (Eds.): Communication for Social Change Anthology: Historical and contemporary readings, South Orange, NJ: CFSC Consortium.

JEREZ, A., LÓPEZ-REY, J. \& SAMPEDRO, V. (2008): Del O'7 a la desobediencia civil: politica e información del movimiento y las ONG de Desarrollo (1994-2000), Madrid: Centro de Investigaciones Sociológicas.

KROHLING PERUZZO, C. M. (2008): "Conceitos de comunicação popular, alternativa e comunitária revisitados: reelaborações no setor", Palabra Clave, 11(2), pp. 367-379.
LENNIE, J. \& TACCHI, J. (2010): “Evaluating communication for development: Trends, challenges and approaches", Draft report on a literature review and consultations conduced for the project: UN Interagency Research, Monitoring and Evaluation Resource Pack for Communication for Development Programs. New York: UNICEF.

LENNIE, J. \& TACCHI, J. (2013): Evaluating communication for development. A framework for social change. London: Routledge.

MANCERA RUEDA, A. \& PANO ALAMÁN, A. (2014): "Las redes sociales como corpus de estudio para el Análisis del discurso mediado por ordenador", Humanidades Digitales. Janus, Anexo 1.

MARÍ-SÁEZ, V. M. \& CEBALLOS-CASTRO, G. (2015): "Análisis bibliométrico sobre "Comunicación, Desarrollo y Cambio Social" en las diez primeras revistas de Comunicación de España", Cuadernos.info, 37, pp. 201-212.

MARÍ-SÁEZ, V. M. (2016): "Communication, development, and social change in Spain: A field between institutionalization and implosion", International Communication Gazette, 78(5), pp. 469-486.

MARÍ-SÁEZ, V. M. (2017): "Historia de la Comunicación para el Desarrollo y el Cambio Social en España. Marginalidad, institucionalización e implosión de un campo en crecimiento", Telos: Cuadernos de comunicación e innovación, 106, pp. 27-38.

MEDA, M. (2012): "Del arte de cambiar para que todo siga igual: el Tercer Sector de la Comunicación y la Ley General Audiovisual en España”, Commons. Revista de Comunicación y Ciudadanía Digital, 1(1), pp. 59-84.

MORAGAS, M. de (2005): "Investigación de la comunicación y política cientifica en España", Paper presented at the Scientific Meeting of the Spanish Society of Journalism. Santiago de Compostela, La Coruña, Spain. 
PARODI, G. (2008): "Lingüística de corpus: una introducción al ámbito", RLA. Revista de lingüística teórica y aplicada, 46(1), pp. 93-119.

PINAZO, D. \& NOS, E. (2013): "Developing moral sensitivity through protest scenarios in international NGDOs' Communication", Communication Research, June 18.

RAYSON， P. \& GARSIDE， R. (2000): "Comparing corpora using frequency profiling", Proceedings of the workshop on Comparing corpora, 9.

SÁNCHEZ-SAUS LASERNA, M. (2017): "Marcadores lingüísticos en comunicación para el desarrollo y el cambio social: principales categorías temáticas en la producción académica sobre el tema", Commons. Revista de Comunicación y Ciudadanía Digital, 6(2), pp. 7898.

SÁNCHEZ-SAUS LASERNA, M. (2018): "Análisis de palabras clave en la comunicación para el desarrollo y el cambio social: el caso de \#comunicambio en Twitter", Culture, Language \& Representation/Cultura, Lenguaje y Representación, 19, pp. 119-139.

SANTOLINO, M. (2010): "Recuperando la esencia: las ONGD como agentes de comunicación para el cambio social", Burgi, T. y Erro, J.
(Coords.): Comunicando para la solidaridad y la cooperación. Cómo salir de la encrucijada, Pamplona: Foro Comunicación, Educación y Ciudadanía, pp. 221-256.

SERVAES, J. (2007a): "Communication for development: Making a difference, a WCCD background study", Servaes, J. (ed.), World Congress on communication for development: Lessons, challenges and the way forward. Washington: The World Bank, pp. 209292.

SERVAES, J. (2007b): "Harnessing the UN system into a common approach on communication for development", Paper prepared for the 10th Inter-Agency Round Table on Communication for Development, Addis Ababa, Ethiopia, 12-14 February.

URANGA, W. (2009): La comunicación comunitaria: proceso cultural, social y politico. Construyendo comunidades. Reflexiones actuales sobre comunicación comunitaria, Buenos Aires: La Crujía, pp. 177186.

WILKINS, K. G. (2000): Redeveloping communication for social change: Theory, practice, and power, Rowman \& Littlefield.

\section{ANEXo}

\begin{tabular}{|c|c|c|c|c|c|c|c|c|c|}
\hline N. ${ }^{\circ}$ & $\begin{array}{c}\text { Frec. } \\
\text { ab- } \\
\text { so- } \\
\text { luta }\end{array}$ & $\begin{array}{l}\text { Frec. } \\
\text { relativa }\end{array}$ & Keyness & Palabra & N. ${ }^{\circ}$ & $\begin{array}{l}\text { Frec. } \\
\text { abs. }\end{array}$ & $\begin{array}{l}\text { Frec. } \\
\text { relativa }\end{array}$ & $\begin{array}{l}\text { Key- } \\
\text { ness }\end{array}$ & Palabra \\
\hline 1 & 1354 & 0,00624622 & 2.759 .843 & social & 76 & 142 & 0,00065507 & 378.758 & recurso \\
\hline 2 & 667 & 0,00307698 & 2.265.709 & $\begin{array}{l}\text { organi- } \\
\text { zación }\end{array}$ & 77 & 165 & 0,00076117 & 376.035 & $\begin{array}{c}\text { desarro- } \\
\text { llar }\end{array}$ \\
\hline 3 & 882 & 0,00406881 & 2.260 .419 & medio & 78 & 77 & 0,00035521 & 375.084 & referir \\
\hline 4 & 1555 & 0,00717347 & 1.675 .187 & $\begin{array}{l}\text { comuni- } \\
\text { cación }\end{array}$ & 79 & 169 & 0,00077962 & 372.588 & mundial \\
\hline 5 & 573 & 0,00264334 & 1.666 .064 & político & 80 & 85 & 0,00039212 & 367.875 & latino \\
\hline 6 & 380 & 0,001753 & 1.602 .439 & ONG & 81 & 147 & 0,00067813 & 362.360 & $\begin{array}{l}\text { coopera- } \\
\text { ción }\end{array}$ \\
\hline 7 & 391 & 0,00180375 & 1.512 .974 & formar & 82 & 172 & 0,00079346 & 357.460 & estrategia \\
\hline
\end{tabular}




\begin{tabular}{|c|c|c|c|c|c|c|c|c|c|}
\hline 8 & 454 & 0,00209438 & 1.468 .070 & derecho & 83 & 88 & 0,00040596 & 350.611 & centrar \\
\hline 9 & 616 & 0,00284171 & 1.314 .024 & público & 84 & 131 & 0,00060432 & 349.662 & dato \\
\hline 10 & 560 & 0,00258337 & 1.270 .787 & nuevo & 85 & 138 & 0,00063662 & 347.159 & teórico \\
\hline 11 & 277 & 0,00127785 & 1.244 .080 & deber & 86 & 202 & 0,00093186 & 346.996 & $\begin{array}{c}\text { interna- } \\
\text { cional }\end{array}$ \\
\hline 12 & 231 & 0,00106564 & 1.144 .125 & $\begin{array}{c}\text { presen- } \\
\text { ciar }\end{array}$ & 87 & 69 & 0,00031831 & 345.551 & $\begin{array}{c}\text { referen- } \\
\text { ciar }\end{array}$ \\
\hline 13 & 594 & 0,00274022 & 1.030 .292 & $\begin{array}{c}\text { desarro- } \\
\text { llo }\end{array}$ & 88 & 124 & 0,00057203 & 343.545 & producir \\
\hline 14 & 416 & 0,00191908 & 894.129 & partir & 89 & 151 & 0,00069659 & 341.330 & mediático \\
\hline 15 & 195 & 0,00089957 & 871.041 & sentir & 90 & 74 & 0,00034137 & 339.718 & solidario \\
\hline 16 & 442 & 0,00203902 & 864.472 & mensaje & 91 & 210 & 0,00096876 & 337.837 & objetivo \\
\hline 17 & 178 & 0,00082114 & 861.383 & unir & 92 & 118 & 0,00054435 & 337.441 & tratar \\
\hline 18 & 198 & 0,00091341 & 779.491 & permitir & 93 & 100 & 0,00046132 & 335.506 & parecer \\
\hline 19 & 183 & 0,00084421 & 763.893 & $\begin{array}{l}\text { relacio- } \\
\text { nar }\end{array}$ & 94 & 73 & 0,00033676 & 334.816 & causar \\
\hline 20 & 304 & 0,0014024 & 753.850 & propio & 95 & 137 & 0,000632 & 327.202 & $\begin{array}{l}\text { institu- } \\
\text { ción }\end{array}$ \\
\hline 21 & 188 & 0,00086727 & 750.140 & resultar & 96 & 115 & 0,00053051 & 325.554 & foro \\
\hline 22 & 226 & 0,00104257 & 736.126 & humano & 97 & 91 & 0,0004198 & 325.311 & medir \\
\hline 23 & 135 & 0,00062278 & 676.077 & privar & 98 & 104 & 0,00047977 & 325.141 & grande \\
\hline 24 & 142 & 0,00065507 & 668.348 & $\begin{array}{c}\text { argen- } \\
\text { tino }\end{array}$ & 99 & 154 & 0,00071043 & 323.527 & persona \\
\hline 25 & 299 & 0,00137934 & 651.924 & acción & 100 & 70 & 0,00032292 & 320.119 & $\begin{array}{c}\text { condicio- } \\
\text { nar }\end{array}$ \\
\hline 26 & 267 & 0,00123171 & 642.397 & dar & 101 & 104 & 0,00047977 & 315.664 & $\begin{array}{l}\text { media- } \\
\text { ción }\end{array}$ \\
\hline 27 & 350 & 0,00161461 & 642.144 & cultural & 102 & 106 & 0489 & 314.805 & presentar \\
\hline 28 & 301 & 0,00138856 & 611.265 & sector & 103 & 230 & 0,00106103 & 312.580 & cambio \\
\hline 29 & 239 & 0,00110255 & 601.524 & $\begin{array}{l}\text { movi- } \\
\text { miento }\end{array}$ & 104 & 81 & 0,00037367 & 311.643 & $\begin{array}{c}\text { cuestio- } \\
\text { nar }\end{array}$ \\
\hline 30 & 352 & 0,00162383 & 597.415 & grupo & 105 & 92 & 0,00042441 & 305.617 & llamar \\
\hline 31 & 145 & 0,00066891 & 567.595 & convertir & 106 & 91 & 0,0004198 & 303.554 & abrir \\
\hline 32 & 246 & 0,00113484 & 559.340 & $\begin{array}{c}\text { econó- } \\
\text { mico }\end{array}$ & 107 & 114 & 0,0005259 & 303.381 & 1levar \\
\hline 33 & 180 & 0,00083037 & 557.315 & tercer & 108 & 63 & 0,00029063 & 297.981 & figurar \\
\hline 34 & 157 & 0,00072427 & 540.595 & entidad & 109 & 67 & 0,00030908 & 295.081 & dedicar \\
\hline 35 & 157 & 0,00072427 & 538.207 & valorar & 110 & 218 & 0,00100567 & 292.721 & modelo \\
\hline 36 & 172 & 0,00079346 & 526.197 & $\begin{array}{l}\text { comuni- } \\
\text { cativo }\end{array}$ & 111 & 122 & 0,00056281 & 291.114 & seguir \\
\hline 37 & 194 & 0,00089495 & 524.409 & primero & 112 & 60 & 0,00027679 & 290.444 & $\begin{array}{l}\text { volunta- } \\
\text { rio }\end{array}$ \\
\hline 38 & 194 & 0,00089495 & 519.996 & $\begin{array}{l}\text { ciuda- } \\
\text { dano }\end{array}$ & 113 & 307 & 0,00141624 & 283.469 & caso \\
\hline 39 & 478 & 0,00220509 & 517.713 & sociedad & 114 & 74 & 0,00034137 & 282.707 & quedar \\
\hline 40 & 366 & 0,00168842 & 516.721 & proceso & 115 & 121 & 0,00055819 & 280.505 & aspecto \\
\hline 41 & 121 & 0,00055819 & 514.105 & diverso & 116 & 83 & 0,00038289 & 280.434 & aparecer \\
\hline 42 & 139 & 0,00064123 & 507.166 & actor & 117 & 604 & 0,00278635 & 280.202 & $\begin{array}{l}\text { informa- } \\
\text { ción }\end{array}$ \\
\hline 43 & 131 & 0,00060432 & 503.161 & distinto & 118 & 69 & 0,00031831 & 279.641 & querer \\
\hline 44 & 191 & 0,00088111 & 498.573 & realizar & 119 & 283 & 0,00130553 & 278.633 & estudio \\
\hline 45 & 124 & 0,00057203 & 497.388 & debatir & 120 & 96 & 0,00044286 & 277.991 & $\begin{array}{l}\text { determi- } \\
\text { nar }\end{array}$ \\
\hline 46 & 226 & 0,00104257 & 494.169 & analizar & 121 & 103 & 0,00047516 & 277.198 & señalar \\
\hline 47 & 102 & 0,00047054 & 484.080 & interesar & 122 & 55 & 0,00025372 & 275.439 & libertar \\
\hline
\end{tabular}




\begin{tabular}{|c|c|c|c|c|c|c|c|c|c|}
\hline 48 & 115 & 0,00053051 & 477.422 & $\begin{array}{c}\text { caracte- } \\
\text { rístico }\end{array}$ & 123 & 132 & 0,00060894 & 273.274 & enfoque \\
\hline 49 & 201 & 0,00092725 & 460.236 & $\begin{array}{l}\text { informa- } \\
\text { tivo }\end{array}$ & 124 & 56 & 0,00025834 & 270.549 & $\begin{array}{l}\text { influen- } \\
\text { ciar }\end{array}$ \\
\hline 50 & 169 & 0,00077962 & 457.634 & $\begin{array}{c}\text { encon- } \\
\text { trar }\end{array}$ & 125 & 150 & 0,00069197 & 269.265 & idea \\
\hline 51 & 328 & 0,00151312 & 457.098 & país & 126 & 60 & 0,00027679 & 266.077 & $\begin{array}{c}\text { concien- } \\
\text { ciar }\end{array}$ \\
\hline 52 & 101 & 0,00046593 & 449.982 & etcétera & 127 & 112 & 0,00051667 & 263.500 & generar \\
\hline 53 & 120 & 0,00055358 & 449.962 & práctico & 128 & 67 & 0,00030908 & 260.062 & vincular \\
\hline 54 & 264 & 0,00121788 & 444.787 & espacio & 129 & 88 & 0,00040596 & 259.529 & $\begin{array}{l}\text { ideoló- } \\
\text { gico }\end{array}$ \\
\hline 55 & 93 & 0,00042902 & 439.554 & temer & 130 & 115 & 0,00053051 & 258.278 & colectivo \\
\hline 56 & 87 & 0,00040135 & 435.694 & mercar & 131 & 86 & 0,00039673 & 256.712 & simbólico \\
\hline 57 & 127 & 0,00058587 & 433.812 & publicar & 132 & 113 & 0,00052129 & 255.673 & sujeto \\
\hline 58 & 171 & 0,00078885 & 431.659 & utilizar & 133 & 66 & 0,00030447 & 255.461 & implicar \\
\hline 59 & 105 & 0,00048438 & 430.218 & $\begin{array}{c}\text { alterna- } \\
\text { tivo }\end{array}$ & 134 & 51 & 0,00023527 & 255.407 & falto \\
\hline 60 & 164 & 0,00075656 & 422.909 & $\begin{array}{c}\text { conside- } \\
\text { rar }\end{array}$ & 135 & 66 & 0,00030447 & 252.311 & surgir \\
\hline 61 & 256 & 0,00118097 & 421.900 & $\begin{array}{c}\text { conte- } \\
\text { nido }\end{array}$ & 136 & 101 & 0,00046593 & 251.765 & definir \\
\hline 62 & 250 & 0,00115329 & 417.730 & año & 137 & 88 & 0,00040596 & 251.095 & $\begin{array}{c}\text { observa- } \\
\text { torio }\end{array}$ \\
\hline 63 & 136 & 0,00062739 & 417.371 & $\begin{array}{c}\text { solidari- } \\
\text { dad }\end{array}$ & 138 & 74 & 0,00034137 & 248.456 & suponer \\
\hline 64 & 161 & 0,00074272 & 414.359 & diferente & 139 & 66 & 0,00030447 & 246.308 & dirigir \\
\hline 65 & 135 & 0,00062278 & 405.898 & ayudar & 140 & 92 & 0,00042441 & 245.189 & incluir \\
\hline 66 & 95 & 0,00043825 & 402.694 & $\begin{array}{l}\text { estruc- } \\
\text { turar }\end{array}$ & 141 & 86 & 0,00039673 & 243.178 & plantear \\
\hline 67 & 133 & 0,00061355 & 397.755 & técnico & 142 & 89 & 0,00041057 & 242.479 & pasar \\
\hline 68 & 99 & 0,0004567 & 394.872 & existir & 143 & 87 & 0,00040135 & 242.354 & $\begin{array}{l}\text { imagina- } \\
\text { rio }\end{array}$ \\
\hline 69 & 178 & 0,00082114 & 392.004 & él & 144 & 66 & 0,00030447 & 237.945 & lógico \\
\hline 70 & 78 & 0,00035983 & 390.622 & $\begin{array}{l}\text { defenso- } \\
\text { ría } \\
\end{array}$ & 145 & 234 & 0,00107948 & 237.718 & sistema \\
\hline 71 & 83 & 0,00038289 & 390.146 & basar & 146 & 60 & 0,00027679 & 237.645 & apuntar \\
\hline 72 & 77 & 0,00035521 & 385.614 & prensar & 147 & 74 & 0,00034137 & 235.596 & experto \\
\hline 73 & 156 & 0,00071965 & 385.269 & elemento & 148 & 71 & 0,00032753 & 235.568 & $\begin{array}{c}\text { mencio- } \\
\text { nar }\end{array}$ \\
\hline 74 & 96 & 0,00044286 & 380.916 & $\begin{array}{l}\text { diferen- } \\
\text { ciar }\end{array}$ & 149 & 113 & 0,00052129 & 234.034 & destacar \\
\hline 75 & 102 & 0,00047054 & 380.129 & $\begin{array}{c}\text { consti- } \\
\text { tuir }\end{array}$ & 150 & 73 & 0,00033676 & 231.404 & $\begin{array}{c}\text { represen- } \\
\text { tar }\end{array}$ \\
\hline
\end{tabular}

Fuente: Elaboración propia

Tabla 3. Primeras 150 palabras clave del corpus académico realmente perteneciente a la CDCS

\begin{tabular}{cccccccccc}
\hline N. $^{\circ}$ & $\begin{array}{c}\text { Frec. } \\
\text { abs. }\end{array}$ & $\begin{array}{c}\text { Frec. } \\
\text { relativa }\end{array}$ & Keyness & Palabra & N. ${ }^{\circ}$ & $\begin{array}{c}\text { Frec. } \\
\text { abs. }\end{array}$ & $\begin{array}{c}\text { Frec. } \\
\text { rel. }\end{array}$ & $\begin{array}{c}\text { Key- } \\
\text { ness }\end{array}$ & Palabra \\
\hline 1 & 873 & 0,00581 & 2498121 & $\begin{array}{c}\text { desarro- } \\
\text { llo }\end{array}$ & 76 & 103 & 0,00069 & 250335 & década \\
\hline 2 & 1460 & 0,00972 & 2249971 & $\begin{array}{c}\text { comuni- } \\
\text { cación }\end{array}$ & 77 & 95 & 0,00063 & 250152 & colectivo \\
\hline 3 & 941 & 0,00626 & 2043377 & social & 78 & 90 & 0,00060 & 247169 & recurso \\
\hline
\end{tabular}




\begin{tabular}{|c|c|c|c|c|c|c|c|c|c|}
\hline 4 & 682 & 0,00454 & 1971276 & medio & 79 & 48 & 0,00032 & 246194 & necesitar \\
\hline 5 & 484 & 0,00322 & 1426110 & cambio & 80 & 109 & 0,00073 & 245373 & mundial \\
\hline 6 & 492 & 0,00327 & 1348272 & nuevo & 81 & 127 & 0,00085 & 242945 & necesario \\
\hline 7 & 410 & 0,00273 & 1301699 & político & 82 & 167 & 0,00111 & 240825 & tecnología \\
\hline 8 & 252 & 0,00168 & 1057129 & formar & 83 & 83 & 0,00055 & 240131 & definir \\
\hline 9 & 199 & 0,00132 & 1004164 & deber & 84 & 80 & 0,00053 & 234472 & vasco \\
\hline 10 & 161 & 0,00107 & 808628 & $\begin{array}{l}\text { alterna- } \\
\text { tivo }\end{array}$ & 85 & 81 & 0,00054 & 234041 & llevar \\
\hline 11 & 153 & 0,00102 & 716931 & $\begin{array}{c}\text { comuni- } \\
\text { tario }\end{array}$ & 86 & 90 & 0,00060 & 230442 & encontrar \\
\hline 12 & 186 & 0,00124 & 709119 & $\begin{array}{l}\text { comuni- } \\
\text { cativo }\end{array}$ & 87 & 104 & 0,00069 & 228830 & persona \\
\hline 13 & 140 & 0,00093 & 702162 & sentir & 88 & 118 & 0,00079 & 223379 & $\begin{array}{l}\text { perspec- } \\
\text { tiva }\end{array}$ \\
\hline 14 & 289 & 0,00192 & 632566 & cultural & 89 & 39 & 0,00026 & 222562 & falta \\
\hline 15 & 114 & 0,00076 & 623019 & unir & 90 & 97 & 0,00065 & 221482 & enfoque \\
\hline 16 & 111 & 0,00074 & 606057 & $\begin{array}{c}\text { intere- } \\
\text { sar }\end{array}$ & 91 & 99 & 0,00066 & 221168 & $\begin{array}{c}\text { desarro- } \\
\text { llar }\end{array}$ \\
\hline 17 & 183 & 0,00122 & 597936 & $\begin{array}{l}\text { ciuda- } \\
\text { dano }\end{array}$ & 92 & 74 & 0,00049 & 218543 & ayudar \\
\hline 18 & 265 & 0,00176 & 575832 & partir & 93 & 130 & 0,00087 & 218162 & acción \\
\hline 19 & 126 & 0,00084 & 563617 & práctico & 94 & 46 & 0,00031 & 218155 & venir \\
\hline 20 & 153 & 0,00102 & 536917 & humano & 95 & 80 & 0,00053 & 216950 & poner \\
\hline 21 & 94 & 0,00063 & 536432 & libertad & 96 & 131 & 0,00087 & 215289 & necesidad \\
\hline 22 & 298 & 0,00198 & 528541 & público & 97 & 43 & 0,00029 & 213905 & demandar \\
\hline 23 & 114 & 0,00076 & 519269 & $\begin{array}{c}\text { partici- } \\
\text { pativo }\end{array}$ & 98 & 145 & 0,00096 & 212357 & espacio \\
\hline 24 & 113 & 0,00075 & 513974 & $\begin{array}{c}\text { relacio- } \\
\text { nar }\end{array}$ & 99 & 113 & 0,00075 & 209962 & teoría \\
\hline 25 & 93 & 0,00062 & 504382 & base & 100 & 179 & 0,00119 & 209508 & publicidad \\
\hline 26 & 209 & 0,00139 & 480123 & radio & 101 & 107 & 0,00071 & 207928 & idea \\
\hline 27 & 236 & 0,00157 & 479435 & modelo & 102 & 61 & 0,00041 & 207349 & sostenible \\
\hline 28 & 83 & 0,00055 & 473658 & mercado & 103 & 86 & 0,00057 & 205463 & considerar \\
\hline 29 & 170 & 0,00113 & 464103 & $\begin{array}{l}\text { movi- } \\
\text { miento }\end{array}$ & 104 & 55 & 0,00037 & 205117 & distinto \\
\hline 30 & 118 & 0,00079 & 463574 & emisora & 105 & 69 & 0,00046 & 204487 & paradigma \\
\hline 31 & 181 & 0,00120 & 453836 & $\begin{array}{l}\text { econó- } \\
\text { mico }\end{array}$ & 106 & 82 & 0,00055 & 203944 & teórico \\
\hline 32 & 97 & 0,00065 & 439012 & debate & 107 & 40 & 0,00027 & 201936 & indigena \\
\hline 33 & 104 & 0,00069 & 438161 & resultar & 108 & 70 & 0,00047 & 201694 & tomar \\
\hline 34 & 271 & 0,00180 & 428526 & proceso & 109 & 65 & 0,00043 & 199713 & nación \\
\hline 35 & 157 & 0,00104 & 413891 & derecho & 110 & 62 & 0,00041 & 198571 & grande \\
\hline 36 & 173 & 0,00115 & 413026 & propio & 111 & 123 & 0,00082 & 198463 & concepto \\
\hline 37 & 246 & 0,00164 & 388496 & pais & 112 & 56 & 0,00037 & 197246 & beneficio \\
\hline 38 & 180 & 0,00120 & 383221 & proyecto & 113 & 108 & 0,00072 & 197211 & $\begin{array}{l}\text { ciudada- } \\
\text { nía }\end{array}$ \\
\hline 39 & 72 & 0,00048 & 380018 & $\begin{array}{l}\text { condi- } \\
\text { cionar }\end{array}$ & 114 & 39 & 0,00026 & 196422 & $\begin{array}{c}\text { compro- } \\
\text { metido }\end{array}$ \\
\hline 40 & 83 & 0,00055 & 377836 & existir & 115 & 55 & 0,00037 & 195989 & bueno \\
\hline 41 & 148 & 0,00098 & 374744 & crítico & 116 & 57 & 0,00038 & 195309 & parecer \\
\hline 42 & 173 & 0,00115 & 365584 & $\begin{array}{c}\text { comuni- } \\
\text { dad }\end{array}$ & 117 & 63 & 0,00042 & 194900 & valorar \\
\hline 43 & 85 & 0,00057 & 354078 & $\begin{array}{c}\text { conver- } \\
\text { tir }\end{array}$ & 118 & 54 & 0,00036 & 194792 & masivo \\
\hline 44 & 162 & 0,00108 & 347762 & local & 119 & 66 & 0,00044 & 194147 & reconocer \\
\hline 45 & 186 & 0,00124 & 347328 & año & 120 & 42 & 0,00028 & 193219 & latino \\
\hline
\end{tabular}




\begin{tabular}{|c|c|c|c|c|c|c|c|c|c|}
\hline 46 & 66 & 0,00044 & 346454 & causa & 121 & 75 & 0,00050 & 193122 & construir \\
\hline 47 & 151 & 0,00100 & 338926 & $\begin{array}{c}\text { pro- } \\
\text { blema }\end{array}$ & 122 & 61 & 0,00041 & 192778 & compartir \\
\hline 48 & 146 & 0,00097 & 328326 & dar & 123 & 127 & 0,00085 & 192401 & objetivo \\
\hline 49 & 235 & 0,00156 & 327205 & cultura & 124 & 77 & 0,00051 & 190030 & mejorar \\
\hline 50 & 61 & 0,00041 & 324248 & temer & 125 & 66 & 0,00044 & 189520 & contribuir \\
\hline 51 & 67 & 0,00045 & 316469 & $\begin{array}{l}\text { estruc- } \\
\text { turar }\end{array}$ & 126 & 43 & 0,00029 & 189109 & querer \\
\hline 52 & 98 & 0,00065 & 308315 & producir & 127 & 87 & 0,00058 & 187688 & mediático \\
\hline 53 & 54 & 0,00036 & 308163 & prensa & 128 & 134 & 0,00089 & 187439 & global \\
\hline 54 & 63 & 0,00042 & 306337 & $\begin{array}{l}\text { disci- } \\
\text { plina }\end{array}$ & 129 & 45 & 0,00030 & 185986 & centrar \\
\hline 55 & 93 & 0,00062 & 301375 & tratar & 130 & 44 & 0,00029 & 185978 & diverso \\
\hline 56 & 66 & 0,00044 & 297599 & surgir & 131 & 82 & 0,00055 & 185888 & comercial \\
\hline 57 & 52 & 0,00035 & 296750 & privar & 132 & 92 & 0,00061 & 184245 & $\begin{array}{c}\text { democra- } \\
\text { cia }\end{array}$ \\
\hline 58 & 78 & 0,00052 & 296722 & permitir & 133 & 59 & 0,00039 & 184001 & justicia \\
\hline 59 & 65 & 0,00043 & 295506 & implicar & 134 & 82 & 0,00055 & 183620 & $\begin{array}{l}\text { globaliza- } \\
\text { ción }\end{array}$ \\
\hline 60 & 142 & 0,00094 & 294965 & $\begin{array}{c}\text { pro- } \\
\text { grama }\end{array}$ & 135 & 62 & 0,00041 & 181951 & pasar \\
\hline 61 & 154 & 0,00102 & 293959 & $\begin{array}{l}\text { partici- } \\
\text { pación }\end{array}$ & 136 & 54 & 0,00036 & 181571 & actor \\
\hline 62 & 295 & 0,00196 & 292690 & sociedad & 137 & 82 & 0,00055 & 180662 & utilizar \\
\hline 63 & 91 & 0,00061 & 285623 & sida & 138 & 33 & 0,00022 & 179418 & referir \\
\hline 64 & 84 & 0,00056 & 282956 & vivir & 139 & 59 & 0,00039 & 178861 & plantear \\
\hline 65 & 89 & 0,00059 & 282224 & señalar & 140 & 83 & 0,00055 & 177780 & popular \\
\hline 66 & 105 & 0,00070 & 281207 & diferente & 141 & 73 & 0,00049 & 177372 & generar \\
\hline 67 & 132 & 0,00088 & 277772 & $\begin{array}{l}\text { organi- } \\
\text { zación }\end{array}$ & 142 & 48 & 0,00032 & 177215 & constituir \\
\hline 68 & 81 & 0,00054 & 273899 & $\begin{array}{l}\text { demo- } \\
\text { crático }\end{array}$ & 143 & 31 & 0,00021 & 176908 & agencia \\
\hline 69 & 55 & 0,00037 & 266863 & $\begin{array}{l}\text { signifi- } \\
\text { car }\end{array}$ & 144 & 31 & 0,00021 & 176908 & $\begin{array}{c}\text { conferen- } \\
\text { cia }\end{array}$ \\
\hline 70 & 75 & 0,00050 & 265689 & ONG & 145 & 77 & 0,00051 & 176846 & $\begin{array}{l}\text { transfor- } \\
\text { mación }\end{array}$ \\
\hline 71 & 142 & 0,00094 & 261188 & $\begin{array}{l}\text { interna- } \\
\text { cional }\end{array}$ & 146 & 65 & 0,00043 & 176784 & alcanzar \\
\hline 72 & 118 & 0,00079 & 259283 & $\begin{array}{c}\text { estrate- } \\
\text { gia }\end{array}$ & 147 & 35 & 0,00023 & 174417 & solidario \\
\hline 73 & 72 & 0,00048 & 258540 & $\begin{array}{c}\text { favore- } \\
\text { cer }\end{array}$ & 148 & 32 & 0,00021 & 173771 & nivel \\
\hline 74 & 106 & 0,00071 & 256613 & $\begin{array}{l}\text { tecnoló- } \\
\text { gico }\end{array}$ & 149 & 87 & 0,00058 & 173016 & $\begin{array}{l}\text { posibili- } \\
\text { dad }\end{array}$ \\
\hline 75 & 88 & 0,00059 & 251941 & masa & 150 & 47 & 0,00031 & 172435 & comenzar \\
\hline
\end{tabular}

Fuente: Sánchez-Saus, 2018.

Listado de revistas y autores con artículos publicados sobre Comunicación, Desarrollo y Cambio Social en las diez primeras revistas españolas en el periodo 2000-2015

\begin{tabular}{|c|c|c|c|c|}
\hline REVISTA & AUTOR & UNIVERSIDAD & VOL & AÑO \\
\hline COMUNICAR & Thomas Tufte & $\begin{array}{l}\text { Universidad de Copenhague, Dina- } \\
\text { marca }\end{array}$ & 26 & 2006 \\
\hline COMUNICAR & $\begin{array}{l}\text { Alejandro Barran- } \\
\text { quero Carretero }\end{array}$ & Universidad de Málaga & 29 & 2007 \\
\hline
\end{tabular}




\begin{tabular}{|c|c|c|c|c|}
\hline COMUNICAR & $\begin{array}{l}\text { Sherri H. Culver y } \\
\text { Thomas Jacobson }\end{array}$ & $\begin{array}{l}\text { Universidad de Filadelfia, Pennsyl- } \\
\text { vania (EEUU) }\end{array}$ & 39 & 2012 \\
\hline $\begin{array}{l}\text { REVISTA } \\
\text { LATINA }\end{array}$ & $\begin{array}{l}\text { Francisco Sierra Ca- } \\
\text { ballero }\end{array}$ & Universidad de Sevilla & 26 & 2000 \\
\hline $\begin{array}{l}\text { REVISTA } \\
\text { LATINA }\end{array}$ & Chiara Sáez Baeza & $\begin{array}{l}\text { Universidad Autónoma de Barce- } \\
\text { lona, UAB }\end{array}$ & 64 & 2009 \\
\hline $\begin{array}{l}\text { REVISTA } \\
\text { LATINA }\end{array}$ & $\begin{array}{l}\text { Txema Ramírez de la } \\
\text { Piscina Martínez }\end{array}$ & Universidad del País Vasco & 65 & 2010 \\
\hline TELOS & José Marques de Melo & $\begin{array}{l}\text { Universidad Metodista Sao Paulo } \\
\text { (Brasil) }\end{array}$ & 51 & 2002 \\
\hline TELOS & $\begin{array}{l}\text { Luis Ramiro Beltrán } \\
\text { Salmón }\end{array}$ & Bolivia & 72 & 2007 \\
\hline TELOS & $\begin{array}{l}\text { Manuel Chaparro Es- } \\
\text { cudero }\end{array}$ & Universidad de Málaga & 74 & 2008 \\
\hline TELOS & $\begin{array}{l}\text { Manuel Chaparro Es- } \\
\text { cudero }\end{array}$ & Universidad de Málaga & 81 & 2009 \\
\hline TELOS & $\begin{array}{l}\text { Manuel Chaparro Es- } \\
\text { cudero }\end{array}$ & Universidad de Málaga & 94 & 2013 \\
\hline $\begin{array}{l}\text { Cuadernos de } \\
\text { Información y } \\
\text { Comunicación } \\
\text { (CIC) }\end{array}$ & Jan Servaes & $\begin{array}{l}\text { Universidad de Massachusetts, Am- } \\
\text { herst (Estados Unidos) }\end{array}$ & 17 & 2012 \\
\hline CIC & Ana Fernández Viso & $\begin{array}{l}\text { Universidad Autónoma de Barce- } \\
\text { lona - Instituto de la Comunicación } \\
\text { de la UAB (InCom-UAB) }\end{array}$ & 17 & 2012 \\
\hline CIC & $\begin{array}{l}\text { Alejandro } \quad \text { Barran- } \\
\text { quero Carretero }\end{array}$ & Universidad Carlos III de Madrid & 17 & 2012 \\
\hline CIC & $\begin{array}{l}\text { Raquel Martínez-Gó- } \\
\text { mez, Pinar Agudiez } \\
\text { Calvo }\end{array}$ & $\begin{array}{l}\text { Universidad Complutense de Ma- } \\
\text { drid }\end{array}$ & 17 & 2012 \\
\hline CIC & $\begin{array}{l}\text { María Cruz Alvarado } \\
\text { López }\end{array}$ & Universidad de Valladolid & 17 & 2012 \\
\hline CIC & $\begin{array}{l}\text { Eloísa Nos, Luís Ama- } \\
\text { dor Iranzo Montés, } \\
\text { Alessandra Farné }\end{array}$ & $\begin{array}{l}\text { Instituto Interuniversitario de Desa- } \\
\text { rrollo Social y Paz (IUDESP) Univer- } \\
\text { sitat Jaume I de Castellón (UJI) }\end{array}$ & 17 & 2012 \\
\hline CIC & $\begin{array}{l}\text { Julio César Herrero, } \\
\text { Ana Toledo Chávarri }\end{array}$ & $\begin{array}{l}\text { Universidad Camilo José Cela (Ma- } \\
\text { drid) }\end{array}$ & 17 & 2012 \\
\hline CIC & Isidoro Arroyo & Universidad Rey Juan Carlos & 18 & 2013 \\
\hline ZER & Víctor Marí & Universidad de Cádiz & 22 & 2007 \\
\hline ÁMBITOS & $\begin{array}{ll}\text { Francisco } & \text { Collado } \\
\text { Campaña } & \end{array}$ & Pablo de Olavide, Sevilla & 17 & 2008 \\
\hline $\begin{array}{l}\text { Comunicación y } \\
\text { Sociedad }\end{array}$ & $\begin{array}{l}\text { Summer Harlow, } \\
\text { Dustin Harp }\end{array}$ & $\begin{array}{l}\text { The University of Texas at Austin. } \\
\text { School of Journalism; The Univer- } \\
\text { sity of Texas at Arlington. Depart- } \\
\text { ment of Communication }\end{array}$ & 26 & 2013 \\
\hline $\begin{array}{l}\text { Estudios sobre } \\
\text { el Mensaje Pe- } \\
\text { riodístico (EMP) }\end{array}$ & $\begin{array}{l}\text { María José Gámez } \\
\text { Fuentes, Eloísa Nos } \\
\text { Aldás }\end{array}$ & Universitat Jaume I, Castellón. & 18 & 2012 \\
\hline EMP & $\begin{array}{l}\text { Concepción Trave- } \\
\text { sedo de Castilla }\end{array}$ & Universidad de Málaga & 19 & 2013 \\
\hline
\end{tabular}

Fuente: Elaboración propia.

Listado de revistas y autores con artículos publicados sobre temáticas afines a la CDCS en las diez primeras revistas españolas en el periodo 2000-2015

\begin{tabular}{lllll}
\hline REVISTA & AUTOR & UNIVERSIDAD & VOL & AÑO \\
\hline ZER & Fernando Trucho & Universidad Rey Juan Carlos & 20 & 2006 \\
\hline
\end{tabular}




\begin{tabular}{|c|c|c|c|c|}
\hline \multirow{2}{*}{ ZER } & $\begin{array}{l}\text { Jose Maria Garcia de } \\
\text { Madariaga }\end{array}$ & Universidad Rey Juan Carlos & 21 & 2006 \\
\hline & $\begin{array}{l}\text { Manuel Javier Callejo } \\
\text { Gallego }\end{array}$ & UNED & 24 & 2008 \\
\hline ZER & $\begin{array}{l}\text { Ariel Jerez Novara; } \\
\text { Jorge Resina de la } \\
\text { Fuente; Ramiro Chico } \\
\text { Hernández }\end{array}$ & $\begin{array}{l}\text { Universidad Complutense de } \\
\text { Madrid; Universidad Complu- } \\
\text { tense de Madrid; Pontificia Uni- } \\
\text { versidad Javeriana }\end{array}$ & 33 & 2012 \\
\hline ZER & $\begin{array}{l}\text { Isidoro Arroyo Alma- } \\
\text { raz; Rebeca Martín } \\
\text { Nieto }\end{array}$ & Universidad Rey Juan Carlos & 31 & 2011 \\
\hline ZER & Isidoro Arroyo & Universidad Rey Juan Carlos & 37 & 2014 \\
\hline $\begin{array}{l}\text { COMUNICA- } \\
\text { CIÓN Y SOCIE- } \\
\text { DAD }\end{array}$ & Daniel H. Cabrera & $\begin{array}{l}\text { Universidad de } \\
\text { Navarra }\end{array}$ & 17 & 2004 \\
\hline $\begin{array}{l}\text { Estudios sobre } \\
\text { el Mensaje Pe- } \\
\text { riodistico (EMP) }\end{array}$ & $\begin{array}{l}\text { Yolanda Martínez So- } \\
\text { lana }\end{array}$ & $\begin{array}{l}\text { Universidad Complutense de } \\
\text { Madrid }\end{array}$ & 13 & 2007 \\
\hline EMP & $\begin{array}{l}\text { Hildegart González } \\
\text { Luis }\end{array}$ & Universidad de Navarra & 19 & 2013 \\
\hline $\begin{array}{l}\text { REVISTA LA- } \\
\text { TINA }\end{array}$ & $\begin{array}{l}\text { Mariano Cebrián He- } \\
\text { rreros }\end{array}$ & $\begin{array}{l}\text { Universidad Complutense de } \\
\text { Madrid }\end{array}$ & 53 & 2003 \\
\hline $\begin{array}{l}\text { REVISTA LA- } \\
\text { TINA }\end{array}$ & $\begin{array}{l}\text { Guillermina Franco, } \\
\text { Carmen Gaona Piso- } \\
\text { nero }\end{array}$ & $\begin{array}{l}\text { Universidad Católica San Anto- } \\
\text { nio de Murcia }\end{array}$ & 56 & 2003 \\
\hline $\begin{array}{l}\text { REVISTA LA- } \\
\text { TINA }\end{array}$ & $\begin{array}{l}\text { Ramón Zallo Elgue- } \\
\text { zabal }\end{array}$ & Universidad del País Vasco & 65 & 2010 \\
\hline $\begin{array}{l}\text { REVISTA LA- } \\
\text { TINA }\end{array}$ & $\begin{array}{l}\text { A Jorge Alonso; R de } \\
\text { Frutos García; E Ga- } \\
\text { larza Fernández }\end{array}$ & Universidad de Málaga & 70 & 2015 \\
\hline $\begin{array}{l}\text { REVISTA LA- } \\
\text { TINA }\end{array}$ & $\begin{array}{l}\text { Isidoro Arroyo Alma- } \\
\text { raz, Miguel Baños } \\
\text { González, Teresa C. } \\
\text { Rodríguez García }\end{array}$ & Universidad Rey Juan Carlos & 64 & 2009 \\
\hline $\begin{array}{l}\text { REVISTA LA- } \\
\text { TINA }\end{array}$ & $\begin{array}{l}\text { Patricia Durán Bravo, } \\
\text { María Belén Fernán- } \\
\text { dez Fuentes }\end{array}$ & $\begin{array}{l}\text { Universidad Autónoma de Pue- } \\
\text { bla, México }\end{array}$ & 65 & 2010 \\
\hline $\begin{array}{l}\text { REVISTA LA- } \\
\text { TINA }\end{array}$ & $\begin{array}{l}\text { Isidoro Arroyo Alma- } \\
\text { raz; M Baños Gonzá- } \\
\text { lez; C Van-Wyck }\end{array}$ & $\begin{array}{l}\text { Universidad Rey Juan Carlos, } \\
\text { Universidad Rey Juan Carlos, } \\
\text { Bournemouth University, Uni- } \\
\text { ted Kingdom }\end{array}$ & 68 & 2013 \\
\hline $\begin{array}{l}\text { REVISTA LA- } \\
\text { TINA }\end{array}$ & $\begin{array}{l}\text { IA Del Amo; A Leta- } \\
\text { mendia; J Diaux }\end{array}$ & Universidad del País Vasco & 69 & 2014 \\
\hline TELOS & Armand Mattelart & & 67 & 2006 \\
\hline TELOS & Eduardo A. Vizer & & 71 & 2007 \\
\hline TELOS & $\begin{array}{l}\text { Luis A. Albornoz y Mi- } \\
\text { cael Herschmann }\end{array}$ & & 72 & 2007 \\
\hline TELOS & $\begin{array}{l}\text { Víctor Manuel Marí } \\
\text { Sáez y Francisco Sie- } \\
\text { rra Caballero }\end{array}$ & Universidad de Sevilla & 74 & 2008 \\
\hline TELOS & Olga Berrios & & 65 & 2005 \\
\hline TELOS & Berta García Orosa & & 69 & 2006 \\
\hline TELOS & $\begin{array}{l}\text { Isidoro Arroyo Alma- } \\
\text { raz, Rebeca Martín } \\
\text { Nieto, Lilia Ivana Ma- } \\
\text { mic }\end{array}$ & $\begin{array}{l}\text { Universidad Rey Juan Carlos; } \\
\text { Universidad Rey Juan Carlos } \\
\text { - }\end{array}$ & 85 & 2010 \\
\hline
\end{tabular}




\begin{tabular}{|c|c|c|c|c|}
\hline COMUNICAR & Sirkku Kotilainen & $\begin{array}{l}\text { Universidad Jyväskylä, Finlan- } \\
\text { dia }\end{array}$ & 32 & 2009 \\
\hline ANÀLISI & Olga del Río Sánchez & $\begin{array}{l}\text { Universitat Autònoma de Barce- } \\
\text { lona }\end{array}$ & 38 & 2009 \\
\hline ÁMBITOS & $\begin{array}{l}\text { Gustavo Adolfo León } \\
\text { Duarte }\end{array}$ & Sonora, México & $7-8$ & 2002 \\
\hline ÁMBITOS & $\begin{array}{l}\text { María del Mar Soria } \\
\text { Ibáñez }\end{array}$ & $\begin{array}{l}\text { Universidad Complutense de } \\
\text { Madrid }\end{array}$ & 27 & 2014 \\
\hline $\begin{array}{l}\text { Cuadernos de } \\
\text { Información } \mathrm{y} \\
\text { Comunicación } \\
\text { (CIC) }\end{array}$ & $\begin{array}{l}\text { Vanesa Saiz Echeza- } \\
\text { rreta }\end{array}$ & $\begin{array}{l}\text { Universidad Complutense de } \\
\text { Madrid }\end{array}$ & 13 & 2008 \\
\hline CIC & Marina Mantini & $\begin{array}{l}\text { Presidenta de la Asociación Cul- } \\
\text { tura Ambiente }\end{array}$ & 17 & 2012 \\
\hline CIC & $\begin{array}{l}\text { Marta Isabel Gonzá- } \\
\text { lez Âlvarez }\end{array}$ & $\begin{array}{l}\text { Doctora en CC de la Informa- } \\
\text { ción, profesora en la U. de la } \\
\text { Rioja y profesional experta en } \\
\text { información y ONGD }\end{array}$ & 17 & 2012 \\
\hline CIC & $\begin{array}{l}\text { María José } \\
\text { Gormazadas } \\
\text { Iráizoz López-Arroba }\end{array}$ & $\begin{array}{l}\text { Universidad Complutense de } \\
\text { Madrid }\end{array}$ & 17 & 2012 \\
\hline
\end{tabular}

Fuente: Elaboración propia. 